\title{
Multi Resident Complex Activity Recognition in Smart Home: A Literature Review
}

\author{
Raihani Mohamed ${ }^{1}$, Thinagaran Perumal ${ }^{2}$, Md Nasir Sulaiman $^{3}$ \\ and Norwati Mustapha ${ }^{4}$ \\ ${ }^{1,2,3,4}$ Department of Computer Science, Faculty of Computer Science and \\ Information Technology, UPM Serdang \\ 1'mraihani@me.com, ${ }^{2}$ thinagaran@upm.edu.my, ${ }^{3}$ nasir@upm.edu.my, \\ ${ }^{4}$ norwati@upm.edu.my
}

\begin{abstract}
This paper presents an overview of state of art of multi resident activity recognition in smart home environment. Generally wearable sensors as well as bespoke sensors are used for tracing the pattern of activity recognition among home dwellers in smart home scenario. Unlike wearable sensor, deployment of bespoke sensors embedded into the environment could be challenging to infer user activities. However, this type of sensors is selected due to human centric concerns, non-obtrusive, inviolate residents' privacy and pervasive concern. Moreover, as human activity is becoming complex when dealing with multi resident, affected that inference activity in smart home scenario are also becoming complicated. Hence, this paper highlight the review of intelligent of smart home including technology sensing involved, previous research on activity recognition area specifically multi resident complex activity recognition in the same environment. We highlighted the multi resident activity recognition including concurrent, interleave and cooperative interaction activity. We present methods behind the main stream of multi resident activity recognition models and algorithms that deploys machine learning as the core subject. Furthermore, this paper also provides potential area for future research.
\end{abstract}

Keywords: complex activity recognition, multi resident, interleave, cooperative, environmental sensor

\section{Introduction}

In recent times, we have seen the emergence of smart objects connecting each other to the physical world. The convergence of sensors and actuators would react automatically to the users within an environment. The sensors are hidden from the user so they become part of the environment, neither require the user to explicitly interact nor wear the devices. Recent research trends evolve not only on the devices and equipment that have "brain" to talk to each other, but to infer on human activity in the environment. The development of ambient intelligent and smart environment is sharing their common goal in supporting and assisting people in activities of daily living (ADL). Smart home in particular, promote the longevity of elderly to stay at home independently, increase the quality of life of home dwellers in term of security and safety. This resulted on research in ambient-assisted living (AAL) such as monitoring of elderly in ADL [1], in early detection of elderly decline in health [2], accessing the elderly functional abilities [3].

Human activity is very complex. Researches in computer science evolve into generalize human activity into real life settings. Methods and approaches developed to achieve the most accurate of human activity [4]. Hence new approaches and algorithms from the sensor based events have also emerged. It takes the sensor fusion readings as inputs and predicts a user's ADL. Smart environment such as building, room and house are well equipped with various sensors including light sensors, motion sensors, 
temperature sensors, etc. The activities like leave house, toileting, showering, sleeping, preparing breakfast, preparing dinner and preparing a beverage are examples of activities chosen based on Katz ADL index, the tool to access cognitive and physical capabilities of elderly person [5]. Some activities identified as simple and some are complex activities. Activities become complex when it involves with many resident in the same environment. Comparing to single resident activity recognition, multi resident activity recognition is more open to interaction on their ADL such as cooperative, concurrent, parallel and interleave activity. Hence, such activity recognition approach is important in real life applications indicates there is a need for activity recognition system with potential approach to support multi resident from discrete binary sensor data in ubiquities technology that supports the AAL in real time. Most of the algorithms are designed to handle one resident in the environment at a time [6]. Hence, not many research available on multi residents in the same environment at a time.

The contributions of this paper are threefold. First, this paper explains on the concept of smart home technology and its relations to human and complex human activity recognition. We also addressed the complexity of multi resident interaction thoroughly. Secondly, this paper aim to summarize the recent methodology in regards to multi resident based activity recognition in smart home as reference. For those who have no background in this area could obtain a comprehensive review on how the experiment is conducted and how the problem is tackled specifically the environmental sensor-based discrete event. Finally, the last section introduces some of the most relevant open problems in the field providing directions for future research.

\section{Related Works}

This section reviews the relationship between smart home technology and human as well as complex activity recognition as well as concept in comprehensive manner.

\subsection{Overview of Smart Home Environment}

In a layman term, the smart home is a residence such as apartment, standalone house equipped with communications network, sensors, domestic appliances and other electronic devices that can be monitored, accessed and controlled to provide services and response accordingly to the needs of users. The technology in smart home environment rapidly evolves from day to day. But, yet the definition of smart home is still elusive. However, from its characteristics for AAL, we can conclude that the smart home is kind of instrumented, interconnected and intelligent in nature. In the early days, the concept was established with the major focus on delivering convenience, improving security and saving energy [7]. Gradually, its functionalities already encompasses entertainment and convenience, safety and health and wellness [8]. Author [9] give in depth illustration on the types of smart home functionalities that forms from smart home.

The increase of demand for smart home technology is related to the potential supports that smart home can provide to the people. Moreover, the interest to support and assist the elderly adults over the age 65 years old is due to the increase of $25 \%$ of the populations predictions in the whole world by 2050 [10]. The elderly adult faces social trends and health care crises due to prevalence of physical, sensory deficiency and cognitive decline among them. This condition already overstretched social and healthcare resources. It increased the resource needs outpace and resource investment [11]. There is need for the nations to prepare necessarily to face the demographic changes of the elderly adult to adjust and allow them to live in best possible conditions. As different people specifically the elderly would adult have different needs, the provision of assistance must be personalized to the individual and tailored based on their necessities. Therefore, the demand on new models for social and healthcare delivery with affordable costs increase and new contribution on community healthcare and social care services are needed [12]. 
Smart home is designed for intelligent service. It includes four key aspects such as the ability of communications between heterogeneous devices in the network, control and manage the system, sensors and actuators that collect information and smart features that can react based on the context environment [9]. Thus, the mentioned capabilities can be used to study on human activity recognition such as early detection of unusual behaviors of elderly for health monitoring purposes [11]. Several smart home projects have been initiated in research labs. Among the latest project are WSN enabled of Smart Home [13], Sweet-Home Project [14], CASAS Smart Home [15] and CareLab [16].

\subsection{Sensor Technology and Human Activity Recognition}

Sensor technology demonstrate the ability to address the challenge of human sensing in smart home environment such as wearable devices, direct into environment components and installed on an existing home infrastructure. The availability of inexpensive sensor and actuator encourage broader implementation in smart home environment. Generally, this requires proper design and implementation based on unique gasp of knowledge that are not limited to a single discipline but in actual an integration aspects of machine learning, decision making, human-machine interface, wireless networking, mobile communication, database, sensor and pervasive computing [17]. Categories of sensors can be classified into vision and non-vision based sensor. The wearable sensor and environmental-based sensor are under non-vision based category. The category is based on the source for raw data collection in activity recognition. Based on the sensor technology, the taxonomy of human activity recognition system can be depicted as in Figure 1.

Example of vision-based sensors is basically inherited from video cameras. It is to observe multiple persons behavior [18], motion capture [19] and monitor environment conditions and changes [20]. The generated sensor data are video sequences or digitized visual data [21]. It can be installed in the fixed places such as the entrance, exit and at any corner based on preferences area of the building, place or rooms. Some of the most popular wearable sensors for activity recognition are accelerometer, temperature, gravity, gyroscope, light, linear acceleration, magnetometer, gyroscope, proximity, humidity and barometer sensor. It is portable, small size and it is often used to inertial measurement and effective to recognize physical movements such as physical exercise, moving directions and etc. The most popular devices are smartphones because it is already pre-equipped with accelerometer, microphone, GPS, barometer etc. [22]. The second category of nonvision based is the environmental sensors. The environmental sensor naturally is low-cost, robust, flexible and data driven intelligent system. It triggers signal data from motion, temperature, door, water, light and state-change sensors to provide feedback to the user. As compare with the wearable sensor that inconvenience in term of ergonomic and user privacy concern, the environmental sensor is non-obtrusive, resilience and human-centric concerns. This case, it is to detect ambient interaction with the environment and suitable to deploy in indoors places such as smart room, intelligent building and smart home. It is divided into two types, the surveillance and activity recognition. We categorized the surveillance such as fire alarm, CCTV and door system for home and building automation system [23]. 


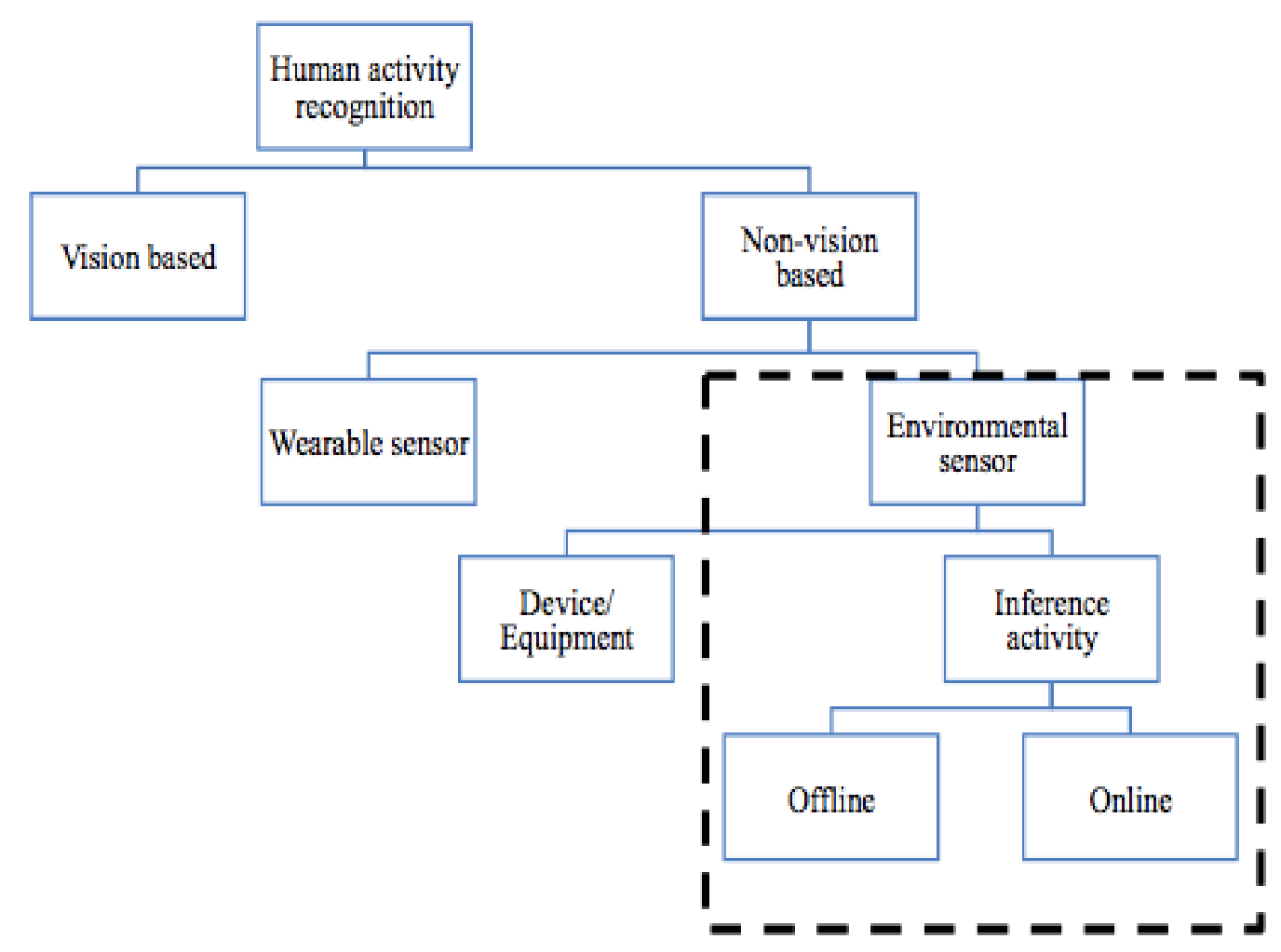

Figure 1. Taxonomy of Human Activity Recognition System in Smart Home

\subsection{Activity Recognition and Activity Modeling}

Activity recognition is key study on human activity recognition to infer the human's activities, action and operation. Activities are composed of cooperative actions or chains of actions. The aims are to recognize the actions and goals of one or more agents from a series of observations on the agents' actions and the environmental conditions. These actions are all related to achieve the overall objective of an activity. Each action has a goal and consists of operations to reach the goal. An operation is a unit component that depends on conditions when the operation is performed [24], [25]. Activity recognition research evolves from activity model and activity theory of state of art.

Activity recognition is the study of inferring the human activities, action and operation. Activities are composed of cooperative actions or chains of actions. The aims are to recognize the actions and goals of one or more agents from a series of observations on the agents' actions and the environmental conditions. These actions are all related to achieve the overall objective of an activity. Each action has a goal and consists of operations to reach the goal. An operation is a unit component that depends on conditions when the operation is performed [24], [25]. Activity recognition research evolves from activity model and activity theory of state of art.

Activity theory is the origin of activity modeling. Historically, a psychologist named L. S. Vygotsk developed the concept of activity theory on 1920s and 1930s. The theory then firstly applied to human-computer interaction (HCI) in the early 1980s as a theoretical framework for HCI design and becoming a benchmark in cross-disciplinary framework in studying different forms of human practices based on its concept and components. The activity theory components consist of four basic structure: subject, tool, objective and outcome [25]. 
Table 1. Hierarchical Level of an Activity with Examples

\begin{tabular}{|l|l|l|}
\hline Levels & Related purpose & Example of purpose \\
\hline Activity & Motive & Completing a software project \\
\hline Action & Goal & Programming related modules \\
\hline Operation & Conditions & Using an operating system \\
\hline
\end{tabular}

Based on the activity theory, the abstractions of things in the real world whether physical or social can be built to model the activity. From the sensor based activity recognition perspective, the activity modeling is a process model to interpret sensor data to infer activities. It is recognized as closely related to the nature and representation of activity models in sequence of situations and emphasis on sequential order. It consider as critical as the process require interpreting the sensor data to infer the activity done by the human especially sensor-based environment.

The general structure of activity recognition consists of two stages that are training and testing that is also called the evaluation stage. The approaches are Data-driven and Knowledge-driven approaches [21]. Having said that, these approaches are essential to determine either the system implementation is real time activity recognition or not. Moreover, additional information from domain knowledge in smart home is essential to infer the activity of its ambient. It can be called as context information that uses to describe the situation of the person in the particular environment. Dey and Abowd [26] propose the definition of context as: "Context is any information that can be used to characterize the situation of entities (i.e., whether a person, place or object) that are considered relevant to the interaction between a user and an application, including the user and the application themselves. Context is typically the location, identity and state of people, groups and computational and physical objects". In more specific way, the big data need to be analyzed in order to provide meaningful information.

Additional information from domain knowledge in smart home is essential to model the activity accurately. The context information used to describe the situation of the person in the particular environment. Context is typically the location, identity and state of people, groups and computational and physical objects" [26]. In more specific way, the context information need to be analyzed in order to provide meaningful information. Real world context such as places, people and things is challenging to infer activity [27]. Bedrooms, living room, bathroom, toilets, kitchen, living room and entrance area are example of places that are specifically assign to the domain smart home. Thus, location information plays an important role in classifying and recognizing ADL [28][29][30]. In this case, we can state that the door, utensils and water tap as the object context for activity to be accomplished. The complexity from context environment need to cater starting from design, development and evolutions phase of the technology applications [27]. Hence, sensors and actuators are the most appropriate choice to cater the problems. The temporal information is important to describe the situation of an activity. Information such as time, duration and location as features can be extracted from sensor data [31][32][33]. Figure 2 shows the types of possible temporal information in smart home. The more features extracted from the data, the more accurate information could be achieved. The temporal information is important to describe the situation of an activity. Information such as time, duration and location as features can be extracted from sensor data [31][32][33]. Figure 2 shows the types of possible temporal information in smart home. The more features extracted from the data, the more accurate information could be achieved. 


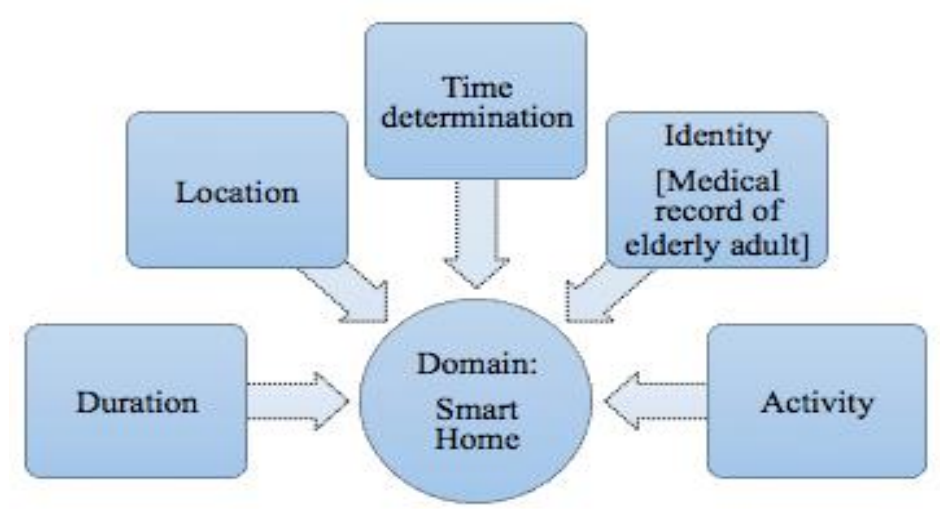

Figure 2. Temporal Information in Smart Home

\subsection{Complex Activity Recognition}

When dealing with the environmental based sensor type installed in the smart home domain, many of research works mainly focus on simplified use of scenarios involving single-user single-activity recognition and single-user multi-activity recognition [21]. However, in real world setting, human activities are always occurs in complex condition. In general, human activities are rather overlapping and concurrent specifically in the smart home environment, there are conditions whereby the activities perform by the residents at the same time in the same place or carry out together in a group [4]. Some are carried out at the same time but in separate location. Table 2 shows the example of details activity types that can be occurred when only dealing with single type of sensor, the environmental sensor in smart home environment.

Table 2. Complex Level of Activity Recognition in Smart Home

\begin{tabular}{|c|c|c|c|c|}
\hline Category & $\begin{array}{l}\text { Activity } \\
\text { Types }\end{array}$ & Mode & Example & Works \\
\hline $\begin{array}{l}\text { Simple } \\
\text { Activity }\end{array}$ & Single & Single & $\begin{array}{l}\text { leave house, go to toilet, go to bed, } \\
\text { filling medication dispenser, hanging } \\
\text { up clothes, read magazine, sweeping } \\
\text { floor, setting the table, watering plants, } \\
\text { prepare dinner, prepare breakfast }\end{array}$ & $\begin{array}{l}{[30],[33]-} \\
{[37]}\end{array}$ \\
\hline \multirow{4}{*}{$\begin{array}{l}\text { Complex } \\
\text { Activity - } \\
\text { People can } \\
\text { do several } \\
\text { activities } \\
\text { at the same } \\
\text { time }\end{array}$} & Concurrent & Single & Watch TV while using phone. & [38] \\
\hline & $\begin{array}{l}\text { Interleave - } \\
\text { intermittent }\end{array}$ & $\begin{array}{l}\text { Single } \\
\& \text { Multi } \\
\text { resident }\end{array}$ & $\begin{array}{l}\text { While cooking, if there is a call from a } \\
\text { friend, resident pause cooking for a } \\
\text { while and after talking to their friend, } \\
\text { they come back to the kitchen and } \\
\text { continue to cook. }\end{array}$ & [39] \\
\hline & Parallel & $\begin{array}{l}\text { Multi } \\
\text { resident }\end{array}$ & $\begin{array}{l}\text { One is toileting and another one is } \\
\text { cooking (at different location but in the } \\
\text { same domain environment) }\end{array}$ & [40], [41] \\
\hline & Cooperative & $\begin{array}{l}\text { Multi } \\
\text { resident }\end{array}$ & $\begin{array}{l}\text { Two ore more residents doing the } \\
\text { same activity that are performed } \\
\text { together in the same location. } \\
\text { E.g.: hanging clothes together }\end{array}$ & {$[42],[43]$} \\
\hline
\end{tabular}

Activities such as concurrent and interleave activity can occur in both single and multiresident situation. However, concurrent and parallel activities differ in term of different location sensor signal. For multi resident, the signal trigger at different location in smart 
home. The interaction between the resident can occur in term of cooperative activity. The cooperative activity only happens whenever there are multi resident in the same environment and the sensor signal at the same location. Figure 3 illustrates examples of cooperative activity in multi resident environment.

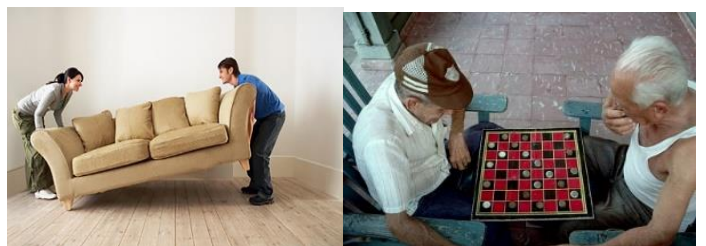

a) Moving Furniture

b) Playing Checkers

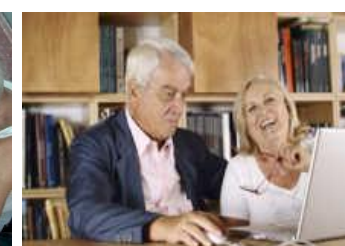

c) Paying Bills

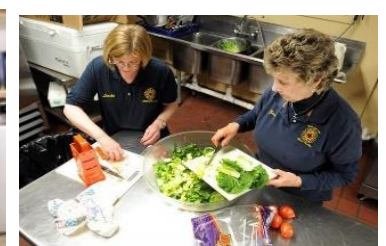

d) Gathering and Packing Picnic Food

Figure 3. Examples of Cooperative Activity of Multi Resident in Smart Home

\section{Multi Resident Current Methodologies and Approach}

Current works on environmental sensor mainly focus activity recognition on single resident is to improve the accuracy activity model on single resident in smart home environment [30], [33]-[37]. Hence, the mention work using the CASAS datasets as their ground of work [44]. In addition, there are also works on multi resident in smart home environment. However, these works focuses on multi-modal sensor that caters the multi resident activity recognition. They model the interaction of multi resident using wearable sensor and environmental sensor. Wang et al. recognize the multi resident activity recognition by adding the RFID wristband reader as resident's wearable sensor [45], [46]. They capture and observe the interaction of the residents using CHMM. Hence the preprocessing stage requires different type of technique due to different type of sensor. There are acceleration data, audio data and RFID data. Tracking the multi resident in smart home easily implemented by using RFID tag that carried by the resident to recognize who is performing what activity. Wilson and Atkeson use RFID key during entrance at the door [47]. They mix the wearable sensor with other type of sensors such as motion detectors, break-beam sensors, pressure mats, and contact switches. This work aims to perform tracking and inference activity recognition for multi resident using bottom-up approach. The information from sensors are then gathered to track the resident by location using Rao-Blackwellished particle filter that is able to handle multiple occupants by performing efficient data association. The works mention above use own data to test the model. Nevertheless, this paper will focus on single type of sensor specifically the environmental sensor for tracking and predicting the activity interaction of multi resident in smart home environment.

Table 3 Comparative Methods from Previous Work

\begin{tabular}{|c|c|c|c|}
\hline Author & Objective and Model & Method & Limitation \\
\hline$[40]$ & $\begin{array}{l}\text { Use simple knowledge in } \\
\text { activities to recognize pattern } \\
\text { and global features what } \\
\text { activity done together \& } \\
\text { individually. }\end{array}$ & $\begin{array}{l}\text { 1. Two-stage } \\
\text { method combine } \\
\text { with HMM \& } \\
\text { CRF }\end{array}$ & $\begin{array}{l}\text { 1.Focus on activity } \\
\text { recognition not the residents. }\end{array}$ \\
\hline [43] & $\begin{array}{l}\text { Propose two types of multi } \\
\text { resident activity models. } \\
\text { Concerning with data } \\
\text { association and interaction } \\
\text { modeling in a multi resident } \\
\text { smart home. }\end{array}$ & $\begin{array}{l}\text { 1.Baseline } \text { Model } \\
\text { Learning using } \\
\text { PHMM and } \\
\text { CHMM } \\
2 . \quad \text { Enhancement } \\
\text { Model Learning }\end{array}$ & $\begin{array}{l}\text { 1. Inflexible model to tackle } \\
\text { increase number of residents. }\end{array}$ \\
\hline
\end{tabular}




\begin{tabular}{|l|l|l|l|}
\hline Author & Objective and Model & Method & Limitation \\
\hline$[42]$ & $\begin{array}{l}\text { Design on interaction } \\
\text { modeling of multi resident by } \\
\text { exploiting auxiliary nodes to } \\
\text { incorporates domain } \\
\text { knowledge and expand the } \\
\text { algorithms to model activities. }\end{array}$ & $\begin{array}{l}\text { 1.PHMM, } \\
\text { CHMM, and extended } \\
\text { from CHMM }\end{array}$ & $\begin{array}{l}\text { 1. Manual label of the } \\
\text { interaction feature maybe } \\
\text { error prone. } \\
\text { 2. Recognize the parallel } \\
\text { activity as single user activity }\end{array}$ \\
\hline$[48]$ & $\begin{array}{l}\text { Enhance model tracking and } \\
\text { recognize the interaction of } \\
\text { cooperative activity using data } \\
\text { association recognizer. }\end{array}$ & $\begin{array}{l}\text { 1. Data association ignores } \\
\text { the interaction among multi } \\
\text { resident. } \\
\text { 2. Lack of preprocessing } \\
\text { technique. }\end{array}$ \\
\hline$[41]$ & $\begin{array}{l}\text { Develop approach that is } \\
\text { effective when activities are } \\
\text { interrupted and interleaved } \\
\text { (individual, individual } \\
\text { requesting assistance, or } \\
\text { cooperative) in parallel, } \\
\text { full/partial in cooperation of } \\
\text { two residents model accuracy. }\end{array}$ & $\begin{array}{l}\text { 1. Develop separate single } \\
\text { model for both residents. } \\
\text { 2. The model not recognizes } \\
\text { interactive and parallel } \\
\text { activities. }\end{array}$ \\
\hline
\end{tabular}

Recent works on multi resident complex activity recognition approach focus on high accurate performance model and some of it discuss on interaction of multi resident in the same environment. Most of the works prefer to use Hidden Markov Model (HMM), Conditional Random Field (CRF), Parallel Hidden Markov Model (PHMM), Coupled Hidden Markov Model (CHMM), interaction-feature enhanced CHMM (ICHMM) and the Dynamic Bayesian Network (DBN) extended from CHMM as their approach. Sensor events from environmental sensor resulted uncertainties and ambiguity to infer human activity models. Thus, the reasons of choosing the probabilistic models because of its ability to represent and reason about variations in the way of an activity may be performed. For example, HMM is designed to determine activities that are most likely equals to an observed sequence of sensor events. Table 3 shows studies using the only environmental sensor and comparisons of each method and approach in details for multi resident using the same ADL Multi Resident Activities datasets from WSU Casas Public Datasets [44]. The datasets are designed with 15 activities involve interaction of cooperative activity and also independent activity carry on in individual and parallel activity. The sensor involve for activity recognition are all environmental sensor types such as motion, door and item sensors.

\subsection{Potential for Future Research}

Sensor technologies indeed give good and positive impact to the smart home applications. Moreover, using only one type of environmental sensor in the environment to predict and recognize the activity of the multi resident in the same environment is essential to address in the future research.

3.1.1. Complex Activity Recognition for Multi Resident: There are conditions whereby the activities perform by the residents at the same time in the same place or carry out together in a group [4]. Activity recognition such as parallel, interleave and cooperation interaction can be occurred in multi user environment. In previous research, they use multi-modal sensor to cater the multi user activity recognition in smart home. Table 3 details the works done regarding the activity recognition in smart environment. However, 
there are limitations and also potential area still open for future research. The cooperative and parallel activity among the multi resident still need area for consideration.

3.1.2. Resident Recognition in Group: Much of the research more focus on the activity recognition, not the residents [40]. It really hard to know which resident is carrying out the activity. Current method approach use data association and manual label on interaction, however poor induced the data will reduce the activity inference. This type of problem gives high challenges for research to apprehend into this area.

3.1.3. Multi Label Classification Model: As mention, current research mostly tackle single user scenario in smart environment. Not much of the research breakthrough the multi resident in the same environment using environmental sensor technology (as mention in Table 3). Once the resident number increase, the model may need to become more sophisticated. Furthermore, adding nodes or edges for two residents will become inflexible as the number of residents increases. Furthermore, the resources needed for training and inference will be extremely demanding when there are numerous vertices and edges in graphical models. Hence, the models need to reflect the multi label classification model in order to incorporate with increasing number of residents in the same domain and recognition of resident identity also can be considered for future research.

\section{Conclusions}

Environmental sensor is pervasive and becoming essential in heterogeneous environment. This has been selected to many reasons and easy implementation without violates human intervention. Thus, human activity recognition is becoming complex when involving multi resident in the same environment. It is a core building block behind the applications. This paper presents a comprehensive survey of the recent advances in activity recognition of multi resident in smart home. We introduce the concepts of multi resident activity recognition, starting with smart home, sensor technology and interaction types. We also review the core machine learning application in the data mining techniques behind the mainstream of multi resident activity recognition.

Hence, multi resident activity recognition is not relatively new area. However, the performance of activity recognition in real life setting require a lot of rooms and efforts in improving its accuracy from existing work as shown in Table 3. The intensive studies in future research can also look into another aspects such as latency problem and conflict resolution techniques. Future algorithms may better explore these aspect. We hope this survey motivates future researchers to devote more attention in interaction and activity recognition in modelling the multi resident actions.

\section{Acknowledgments}

The work is supported by IPM Putra Research Grant Scheme (Vote Number: 9437200) funded by Ministry of Education, Malaysia.

\section{References}

[1] T. Tsukiyama, "In-home Health Monitoring System for Solitary Elderly", Procedia Comput. Sci., vol. 63, pp. 229-235, 2015.

[2] M. L. Lee, "Embedded assessment of wellness with smart home sensors," 12th ACM Int. Conf. Adjun. Pap. Ubiquitous Comput., p. 473, 2010.

[3] S. Mahmoud, A. Lotfi, and C. Langensiepen, "Behavioural pattern identification and prediction in intelligent environments,” Appl. Soft Comput. J., vol. 13, no. 4, pp. 1813-1822, 2013.

[4] E. Kim, S. Helal, and D. Cook, "Human Activity Recognition and Pattern Discovery," Pervasive Comput. IEEE, vol. 9, no. 1, pp. 48-53, 2010.

[5] S. Katz, T. D. Downs, H. R. Cash, and R. C. Grotz, "Progress in Development of the Index of ADL," 
Gerontologist, vol. 10, no. 1 Part 1, pp. 20-30, Mar. 1970.

[6] A. S. Crandall and D. J. Cook, "Coping with multiple residents in a smart environment," J. Ambient Intell. Smart Environ., vol. 1, no. 4, pp. 323-334, 2009.

[7] D. Ding, R. a. Cooper, P. F. Pasquina, and L. Fici-Pasquina, "Sensor technology for smart homes," Maturitas, vol. 69, no. 2, pp. 131-136, 2011.

[8] L. C. De Silva, C. Morikawa, and I. M. Petra, "State of the art of smart homes," Eng. Appl. Artif. Intell., vol. 25, no. 7, pp. 1313-1321, 2012.

[9] N. Balta-Ozkan, R. Davidson, M. Bicket, and L. Whitmarsh, "The development of smart homes market in the UK," Energy, vol. 60, pp. 361-372, 2013.

[10] United Nations: Department of Social and Economic Affairs, "World population prospects: The 2012 revision, DVD edition," Popul. Div. 2013, 2013.

[11] G. Paré, M. Jaana, and C. Sicotte, "Systematic Review of Home Telemonitoring for Chronic Diseases: The Evidence Base," J. Am. Med. Informatics Assoc., vol. 14, no. 3, pp. 269-277, 2007.

[12] C. Ham, A. Dixon, and B. Brooke, "Transforming the Delivery of Health and Social Care: The case for fundament change," pp. 1-52, 2012.

[13] N. K. Suryadevara, S. C. Mukhopadhyay, R. Wang, and R. K. Rayudu, "Forecasting the behavior of an elderly using wireless sensors data in a smart home,” Eng. Appl. Artif. Intell., vol. 26, no. 10, pp. 26412652, 2013.

[14] F. Portet, M. Vacher, C. Golanski, C. Roux, and B. Meillon, "Design and evaluation of a smart home voice interface for the elderly: Acceptability and objection aspects,” Pers. Ubiquitous Comput., vol. 17, no. 1, pp. 127-144, 2013.

[15] D. J. Cook, A. S. Crandall, B. L. Thomas, and N. C. Krishnan, "CASAS: A smart home in a box," Computer (Long. Beach. Calif)., vol. 46, no. 7, pp. 62-69, 2013.

[16] B. de Ruyter and E. Pelgrim, “Ambient Assisted-Living Reserach in CareLab,” Interactions, pp. 30-33, 2007.

[17] D. J. Cook and M. Youngblood, "Smart Homes,” Encycl. Human-Computer Interact., 2004.

[18] T. Kaneko, M. Shimosaka, S. Odashima, R. Fukui, and T. Sato, "A fully connected model for consistent collective activity recognition in videos," Pattern Recognit. Lett., vol. 43, no. 1, pp. 109-118, 2014.

[19] E. Fotiadou and N. Nikolaidis, "Activity-based methods for person recognition in motion capture sequences,” Pattern Recognit. Lett., vol. 49, pp. 48-54, 2014.

[20] M. Ziaeefard and R. Bergevin, "Semantic human activity recognition: A literature review," Pattern Recognit., vol. 48, no. 8, pp. 2329-2345, 2015.

[21] L. Chen, J. Hoey, C. D. Nugent, D. J. Cook, and Z. Yu, "Sensor-based activity recognition," IEEE Trans. Syst. Man Cybern. Part C Appl. Rev., vol. 42, no. 6, pp. 790-808, 2012.

[22] X. Su, H. Tong, and P. Ji, “Activity recognition with smartphone sensors," Tsinghua Sci. Technol., vol. 19, no. 3, pp. 235-249, 2014

[23] T. Perumal, N. Sulaiman, N. Mustapha, A. Shahi, and R. Thinaharan, "Proactive Architecture for Internet of Things ( IoTs ) Management in Smart Homes," pp. 16-17, 2014.

[24] E. Kim and S. Helal, "Revisiting Human Activity Frameworks," Sens. Syst. Softw., no. 5, pp. 219-234, 2011.

[25] K. Kuutti, “Activity theory as a potential framework for human-computer interaction research," pp. 17 44, Dec. 1995.

[26] A. K. Dey and G. D. Abowd, "Towards a Better Understanding of Context and Context-Awareness," Comput. Syst., vol. 40, no. 3, pp. 304-307, 1999.

[27] A. Dey, G. Abowd, and D. Salber, "A Conceptual Framework and a Toolkit for Supporting the Rapid Prototyping of Context-Aware Applications,” Human-Computer Interact., vol. 16, no. 2, pp. 97-166, 2001.

[28] A. Fleury, M. Vacher, and N. Noury, "SVM-based multimodal classification of activities of daily living in health smart homes: Sensors, algorithms, and first experimental results," IEEE Trans. Inf. Technol. Biomed., vol. 14, no. 2, pp. 274-283, 2010.

[29] G. Okeyo, L. Chen, H. Wang, and R. Sterritt, "Dynamic sensor data segmentation for real-time knowledge-driven activity recognition," Pervasive Mob. Comput., vol. 10, pp. 155-172, 2014.

[30] J. Wan, M. J. O'Grady, and G. M. P. O'Hare, "Dynamic sensor event segmentation for real-time activity recognition in a smart home context," Pers. Ubiquitous Comput., vol. 19, no. 2, pp. 287-301, 2014.

[31] S. McKeever, J. Ye, L. Coyle, C. Bleakley, and S. Dobson, "Activity recognition using temporal evidence theory," J. Ambient Intell. Smart Environ., vol. 2, no. 3, pp. 253-269, 2010.

[32] V. Jakkula, a Crandall, and D. Cook, "Enhancing anomaly detection using temporal pattern discovery," Adv. Intell. Environ., pp. 175-194, 2009.

[33] V. R. Jakkula, A. S. Crandall, and D. J. Cook, "Knowledge discovery in entity based smart environment resident data using temporal relation based data mining," Proc. - IEEE Int. Conf. Data Mining, ICDM, pp. 625-630, 2007.

[34] P. Rashidi, D. J. Cook, L. B. Holder, and M. Schmitter-Edgecombe, "Discovering Activities to Recognize and Track in a Smart Environment," IEEE Trans. Knowl. Data Eng., vol. 23, no. 4, pp. 527539, Apr. 2011

[35] L. G. Fahad, A. Ali, and M. Rajarajan, "Long term analysis of daily activities in a smart home," Eur. 
Symp. Artif. Neural Networks, Comput. Intell. Mach. Learn., no. April, pp. 419 - 424, 2013.

[36] D. J. Cook, "Learning Setting- Activity Models for Smart Spaces," IEEE Intell. Syst., pp. 32-38, 2012.

[37] P. Rashidi and D. J. Cook, "Activity knowledge transfer in smart environments," Pervasive Mob. Comput., vol. 7, no. 3, pp. 331-343, 2011.

[38] B. Das, C. Chen, a Seelye, and D. Cook, "An automated propmting system for smart environments," Int. Conf. Smart Homes Heal. Telemat., pp. 9-16, 2011.

[39] M. E. Pollack, L. Brown, D. Colbry, C. E. McCarthy, C. Orosz, B. Peintner, S. Ramakrishnan, and I. Tsamardinos, "Autominder: An intelligent cognitive orthotic system for people with memory impairment," in Robotics and Autonomous Systems, 2003, vol. 44, no. 3-4, pp. 273-282.

[40] R. Chen and Y. Tong, "A Two-stage Method for Solving Multi-resident Activity Recognition in Smart Environments," Entropy, vol. 16, no. 4, pp. 2184-2203, 2014.

[41] G. Singla, D. J. Cook, and M. Schmitter-Edgecombe, "Recognizing independent and joint activities among multiple residents in smart environments," J. Ambient Intell. Humaniz. Comput., vol. 1, no. 1, pp. 57-63, 2010.

[42] Y. T. Chiang, K. C. Hsu, C. H. Lu, L. C. Fu, and J. Y. J. Hsu, "Interaction models for multiple-resident activity recognition in a smart home,” IEEE/RSJ 2010 Int. Conf. Intell. Robot. Syst. IROS 2010 - Conf. Proc., pp. 3753-3758, 2010.

[43] C. H. Lu and Y. T. Chiang, "Interaction-feature enhanced multiuser model learning for a home environment using ambient sensors," Int. J. Intell. Syst., vol. 29, no. 11, pp. 1015-1046, 2014.

[44] "Datasets | WSU CASAS on WordPress.com." [Online]. Available: http://ailab.wsu.edu/casas/datasets.html. [Accessed: 25-Dec-2015].

[45] L. Wang, T. Gu, X. Tao, and J. Lu, "Sensor-based human activity recognition in a multi-user scenario," in Lecture Notes in Computer Science (including subseries Lecture Notes in Artificial Intelligence and Lecture Notes in Bioinformatics), 2009, vol. 5859 LNCS, pp. 78-87.

[46] L. Wang, T. Gu, X. Tao, H. Chen, and J. Lu, "Multi-User Activity Recognition in a Smart Home," Act. Recognit. Pervasive Intell. Environ., vol. 4, pp. 59-81, 2011.

[47] D. Wilson and C. Atkeson, "Simultaneous tracking and activity recognition (STAR) using many anonymous, binary sensors," Proc. Third Int. Conf. Pervasie Comput., vol. 3468, pp. 62-79, 2005.

[48] K. C. Hsu, Y. T. Chiang, G. Y. Lin, C. H. Lu, J. Y. J. Hsu, and L. C. Fu, "Strategies for Inference Mechanism of Conditional Random Fields for Multiple-Resident Activity Recognition in a Smart Home,” Trends Appl. Intell. Syst. Pt I, Proc., vol. 6096, pp. 417-426, 2010.

Authors

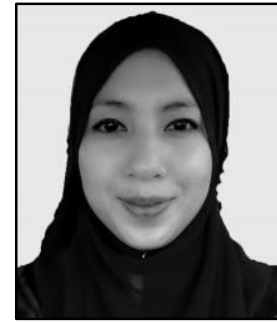

Raihani Mohamed received her B. Science (Management Information Systems) in 2003 at Kulliyyah of ICT at International Islamic University Malaysia. She completed her MSc. degree in Information Technology at Faculty of Computer and Mathematical Sciences at University of Technology MARA. Her main interests are smart home systems, Ambient Assisted Living Environment, machine learning technique and human activity recognition.

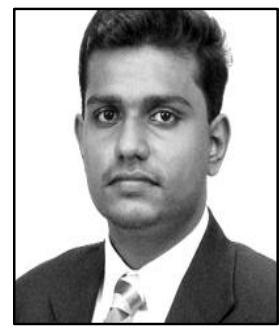

Dr. Thinagaran Perumal received his B.Eng. in 2003 at Department of Computer and Communication Systems Engineering, Universiti Putra Malaysia. He completed his M.Sc. degree in Intelligent Systems and $\mathrm{PhD}$ in Smart Technology and Robotics at Institute of Advanced Technology, Universiti Putra Malaysia. His main interests are smart home systems, middleware technologies and embedded system design. He is a Senior Member of IEEE.

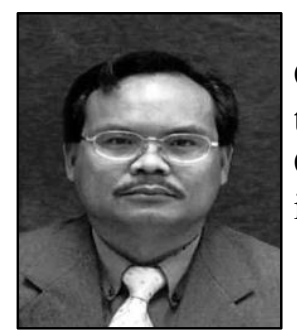

Assoc. Prof. Dr. Md Nasir Sulaiman obtained his $\mathrm{PhD}$ in Computer Science from Loughborough University of Technology in the United Kingdom. He is currently an associate professor of Computer Science at Universiti Putra Malaysia. His research interests include intelligent computing, data mining and machine learning. 


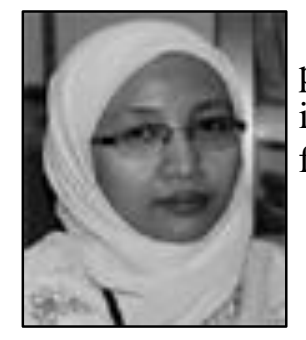

Assoc. Prof. Dr. Norwati Mustapha is currently associate professor of Computer Science in UPM. Her research interests include data mining and machine learning. She is now Deputy Dean for Academics in the faculty. 